\title{
Invertibility of Matrices of Field Elements
}

\author{
Yatsuka Nakamura \\ Shinshu University \\ Nagano, Japan
}

\author{
Kunio Oniumi \\ Shinshu University \\ Nagano, Japan
}

\author{
Wenpai Chang \\ Nan Kai Institute of Technology \\ Nantou County, Taiwan
}

\begin{abstract}
Summary. In this paper the theory of invertibility of matrices of field elements (see e.g. [5], [6]) is developed. The main purpose of this article is to prove that the left invertibility and the right invertibility are equivalent for a matrix of field elements. To prove this, we introduced a special transformation of matrix to some canonical forms. Other concepts as zero vector and base vectors of field elements are also introduced as a preparation.
\end{abstract}

MML identifier: MATRIX14, version: $\underline{7.9 .01 \quad 4.101 .1015}$

The papers [14], [3], [7], [17], [4], [13], [15], [10], [1], [12], [18], [16], [9], [8], [2], and [11] provide the terminology and notation for this paper.

\section{Preliminaries}

We use the following convention: $x, y$ denote sets, $n, m, i, j$ denote elements of $\mathbb{N}$, and $K$ denotes a field.

Let $K$ be a non empty zero structure and let us consider $n$. The functor $0_{K}^{n}$ yields a finite sequence of elements of $K$ and is defined by:

(Def. 1) $0_{K}^{n}=n \mapsto 0_{K}$.

Let $K$ be a non empty zero structure and let us consider $n$. Then $0_{K}^{n}$ is an element of (the carrier of $K)^{n}$.

In the sequel $L$ denotes a non empty additive loop structure.

The following three propositions are true: 
(1) Every finite sequence $x$ of elements of $L$ is an element of (the carrier of $L)^{\operatorname{len} x}$.

(2) For all finite sequences $x_{1}, x_{2}$ of elements of $L$ such that len $x_{1}=$ len $x_{2}$ holds len $\left(x_{1}+x_{2}\right)=\operatorname{len} x_{1}$.

(3) For all finite sequences $x_{1}, x_{2}$ of elements of $L$ such that len $x_{1}=$ len $x_{2}$ holds len $\left(x_{1}-x_{2}\right)=$ len $x_{1}$.

In the sequel $G$ is a non empty multiplicative loop structure.

Next we state four propositions:

(4) Let $x_{1}, x_{2}$ be finite sequences of elements of $G$ and given $i$. If $i \in \operatorname{dom}\left(x_{1} \bullet\right.$ $\left.x_{2}\right)$, then $\left(x_{1} \bullet x_{2}\right)(i)=\left(x_{1}\right)_{i} \cdot\left(x_{2}\right)_{i}$ and $\left(x_{1} \bullet x_{2}\right)_{i}=\left(x_{1}\right)_{i} \cdot\left(x_{2}\right)_{i}$.

(5) Let $x_{1}, x_{2}$ be finite sequences of elements of $L$ and $i$ be a natural number. If len $x_{1}=$ len $x_{2}$ and $1 \leq i \leq$ len $x_{1}$, then $\left(x_{1}+x_{2}\right)(i)=\left(x_{1}\right)_{i}+\left(x_{2}\right)_{i}$ and $\left(x_{1}-x_{2}\right)(i)=\left(x_{1}\right)_{i}-\left(x_{2}\right)_{i}$.

(6) For every element $a$ of $K$ and for every finite sequence $x$ of elements of $K$ holds $-a \cdot x=(-a) \cdot x$ and $-a \cdot x=a \cdot-x$.

(7) For all finite sequences $x_{1}, x_{2}, y_{1}, y_{2}$ of elements of $G$ such that len $x_{1}=$ len $x_{2}$ and len $y_{1}=$ len $y_{2}$ holds $x_{1} \frown y_{1} \bullet x_{2} \frown y_{2}=\left(x_{1} \bullet x_{2}\right)^{\frown}\left(y_{1} \bullet y_{2}\right)$.

Let us consider $K$ and let $e_{1}, e_{2}$ be finite sequences of elements of $K$. We introduce $\left|\left(e_{1}, e_{2}\right)\right|$ as a synonym of $e_{1} \cdot e_{2}$.

Next we state several propositions:

(8) Let $x, y$ be finite sequences of elements of $K$ and $a$ be an element of $K$. If len $x=$ len $y$, then $a \cdot x \bullet y=a \cdot(x \bullet y)$ and $x \bullet a \cdot y=a \cdot(x \bullet y)$.

(9) For all finite sequences $x, y$ of elements of $K$ and for every element $a$ of $K$ such that len $x=\operatorname{len} y$ holds $|(a \cdot x, y)|=a \cdot|(x, y)|$.

(10) For all finite sequences $x, y$ of elements of $K$ and for every element $a$ of $K$ such that len $x=\operatorname{len} y$ holds $|(x, a \cdot y)|=a \cdot|(x, y)|$.

(11) Let $x, y_{1}, y_{2}$ be finite sequences of elements of $K$ and $a$ be an element of $K$. If len $x=\operatorname{len} y_{1}$ and len $x=\operatorname{len} y_{2}$, then $\left|\left(x, y_{1}+y_{2}\right)\right|=\left|\left(x, y_{1}\right)\right|+$ $\left|\left(x, y_{2}\right)\right|$.

(12) For all finite sequences $x_{1}, x_{2}, y_{1}, y_{2}$ of elements of $K$ such that len $x_{1}=$ len $x_{2}$ and len $y_{1}=$ len $y_{2}$ holds $\left|\left(x_{1} \frown y_{1}, x_{2} \frown y_{2}\right)\right|=\left|\left(x_{1}, x_{2}\right)\right|+\left|\left(y_{1}, y_{2}\right)\right|$.

(13) For every element $p_{1}$ of (the carrier of $\left.K\right)^{n}$ holds $p_{1} \bullet n \mapsto 0_{K}=n \mapsto 0_{K}$.

Let us consider $n$, let us consider $K$, and let $A$ be a square matrix over $K$ of dimension $n$. We introduce Inv $A$ as a synonym of $A^{\smile}$.

\section{Zero Vector and Base Vectors of Field Elements}

Next we state several propositions:

$$
I_{K}^{0 \times 0}=0_{K}^{0 \times 0} \text { and } I_{K}^{0 \times 0}=\emptyset .
$$


(15) For every square matrix $A$ over $K$ of dimension 0 holds $A=\emptyset$ and $A=I_{K}^{0 \times 0}$ and $A=0_{K}^{0 \times 0}$.

(16) Every square matrix over $K$ of dimension 0 is invertible.

(17) For all square matrices $A, B, C$ over $K$ of dimension $n$ holds $(A \cdot B) \cdot C=$ $A \cdot(B \cdot C)$.

(18) Let $A, B$ be square matrices over $K$ of dimension $n$. Then $A$ is invertible and $B=A^{\smile}$ if and only if $B \cdot A=I_{K}^{n \times n}$ and $A \cdot B=I_{K}^{n \times n}$.

(19) Let $A$ be a square matrix over $K$ of dimension $n$. Then $A$ is invertible if and only if there exists a square matrix $B$ over $K$ of dimension $n$ such that $B \cdot A=I_{K}^{n \times n}$ and $A \cdot B=I_{K}^{n \times n}$.

(20) For every finite sequence $x$ of elements of $K$ holds $\left|\left(x, 0_{K}^{\text {len } x}\right)\right|=0_{K}$.

(21) For every finite sequence $x$ of elements of $K$ holds $\left|\left(0_{K}^{\operatorname{len} x}, x\right)\right|=0_{K}$.

(22) For every element $a$ of $K$ holds $\left|\left(\left\langle 0_{K}\right\rangle,\langle a\rangle\right)\right|=0_{K}$.

Let $K$ be a non empty set, let $n$ be a natural number, and let $a$ be an element of $K$. Then $n \mapsto a$ is a finite sequence of elements of $K$.

Let us consider $K$ and let $n, i$ be natural numbers. The $i$-versor in $K^{n}$ yields a finite sequence of elements of $K$ and is defined by:

(Def. 2) The $i$-versor in $K^{n}=\operatorname{Replace}\left(n \mapsto 0_{K}, i, 1_{K}\right)$.

Next we state several propositions:

(23) For all natural numbers $n, i$ holds len (the $i$-versor in $\left.K^{n}\right)=n$.

(24) For all natural numbers $i, n$ such that $1 \leq i \leq n$ holds (the $i$-versor in $\left.K^{n}\right)(i)=1_{K}$.

(25) Let $i, j, n$ be natural numbers. Suppose $1 \leq i \leq n$ and $1 \leq j \leq n$ and $i \neq j$. Then (the $i$-versor in $\left.K^{n}\right)(j)=0_{K}$.

(26) For all natural numbers $i, n$ such that $1 \leq i \leq n$ holds $I_{K}^{n \times n}(i)=$ the $i$-versor in $K^{n}$.

(27) For all $i, j$ such that $1 \leq i \leq n$ and $1 \leq j \leq n$ holds $I_{K}^{n \times n}{ }_{i, j}=$ (the $i$-versor in $\left.K^{n}\right)(j)$.

(28) Let $A$ be a square matrix over $K$ of dimension $n$. Then $A=0_{K}^{n \times n}$ if and only if for all elements $i, j$ of $\mathbb{N}$ such that $1 \leq i \leq n$ and $1 \leq j \leq n$ holds $A_{i, j}=0_{K}$.

(29) Let $A$ be a square matrix over $K$ of dimension $n$. Then $A=I_{K}^{n \times n}$ if and only if for all elements $i, j$ of $\mathbb{N}$ such that $1 \leq i \leq n$ and $1 \leq j \leq n$ holds $A_{i, j}=\left(i=j \rightarrow 1_{K}, 0_{K}\right)$. 


\section{Conditions of Invertibility}

One can prove the following propositions:

(30) For all square matrices $A, B$ over $K$ of dimension $n$ holds $(A \cdot B)^{\mathrm{T}}=$ $B^{\mathrm{T}} \cdot A^{\mathrm{T}}$.

(31) For every square matrix $A$ over $K$ of dimension $n$ such that $A$ is invertible holds $A^{\mathrm{T}}$ is invertible and $\left(A^{\mathrm{T}}\right)^{\smile}=\left(A^{\smile}\right)^{\mathrm{T}}$.

(32) Let $x$ be a finite sequence of elements of $K$ and $a$ be an element of $K$. Given $i$ such that $1 \leq i \leq \operatorname{len} x$ and $x(i)=a$ and for every $j$ such that $j \neq i$ and $1 \leq j \leq$ len $x$ holds $x(j)=0_{K}$. Then $\sum x=a$.

(33) Let $f_{1}, f_{2}$ be finite sequences of elements of $K$. Then $\operatorname{dom}\left(f_{1} \bullet f_{2}\right)=$ $\operatorname{dom} f_{1} \cap \operatorname{dom} f_{2}$ and for every $i$ such that $i \in \operatorname{dom}\left(f_{1} \bullet f_{2}\right)$ holds $\left(f_{1} \bullet f_{2}\right)(i)=$ $\left(f_{1}\right)_{i} \cdot\left(f_{2}\right)_{i}$.

(34) Let $x, y$ be finite sequences of elements of $K$ and given $i$. Suppose len $x=$ $m$ and $y=x \bullet$ the $i$-versor in $K^{m}$ and $1 \leq i \leq m$. Then $y(i)=x(i)$ and for every $j$ such that $j \neq i$ and $1 \leq j \leq m$ holds $y(j)=0_{K}$.

(35) Let $x$ be a finite sequence of elements of $K$. Suppose len $x=m$ and $1 \leq i \leq m$. Then $\mid\left(x\right.$, the $i$-versor in $\left.K^{m}\right) \mid=x(i)$ and $\mid(x$, the $i$-versor in $\left.K^{m}\right) \mid=x_{i}$.

(36) For all $m, i$ such that $1 \leq i \leq m$ holds |(the $i$-versor in $K^{m}$, the $i$-versor in $\left.K^{m}\right) \mid=1_{K}$.

(37) Let $a$ be an element of $K$ and $P, Q$ be square matrices over $K$ of dimension $n$. Suppose that $n>0$ and $a \neq 0_{K}$ and $P_{1,1}=a^{-1}$ and for every $i$ such that $1<i \leq n$ holds $P(i)=$ the $i$-versor in $K^{n}$ and $Q_{1,1}=a$ and for every $j$ such that $1<j \leq n$ holds $Q_{1, j}=-a \cdot P_{1, j}$ and for every $i$ such that $1<i \leq n$ holds $Q(i)=$ the $i$-versor in $K^{n}$. Then $P$ is invertible and $Q=P^{\smile}$.

(38) Let $a$ be an element of $K$ and $P$ be a square matrix over $K$ of dimension $n$. Suppose $n>0$ and $a \neq 0_{K}$ and $P_{1,1}=a^{-1}$ and for every $i$ such that $1<i \leq n$ holds $P(i)=$ the $i$-versor in $K^{n}$. Then $P$ is invertible.

(39) Let $A$ be a square matrix over $K$ of dimension $n$. Suppose $n>0$ and $A_{1,1} \neq 0_{K}$. Then there exists a square matrix $P$ over $K$ of dimension $n$ such that

(i) $P$ is invertible,

(ii) $(A \cdot P)_{1,1}=1_{K}$,

(iii) for every $j$ such that $1<j \leq n$ holds $(A \cdot P)_{1, j}=0_{K}$, and

(iv) for every $i$ such that $1<i \leq n$ and $A_{i, 1}=0_{K}$ holds $(A \cdot P)_{i, 1}=0_{K}$.

(40) Let $A$ be a square matrix over $K$ of dimension $n$. Suppose $n>0$ and $A_{1,1} \neq 0_{K}$. Then there exists a square matrix $P$ over $K$ of dimension $n$ such that 
(i) $P$ is invertible,

(ii) $(P \cdot A)_{1,1}=1_{K}$,

(iii) for every $i$ such that $1<i \leq n$ holds $(P \cdot A)_{i, 1}=0_{K}$, and

(iv) for every $j$ such that $1<j \leq n$ and $A_{1, j}=0_{K}$ holds $(P \cdot A)_{1, j}=0_{K}$.

(41) Let $A$ be a square matrix over $K$ of dimension $n$. Suppose $n>0$ and $A_{1,1} \neq 0_{K}$. Then there exist square matrices $P, Q$ over $K$ of dimension $n$ such that

(i) $P$ is invertible,

(ii) $Q$ is invertible,

(iii) $(P \cdot A \cdot Q)_{1,1}=1_{K}$,

(iv) for every $i$ such that $1<i \leq n$ holds $(P \cdot A \cdot Q)_{i, 1}=0_{K}$, and

(v) for every $j$ such that $1<j \leq n$ holds $(P \cdot A \cdot Q)_{1, j}=0_{K}$.

\section{A Transformation of Matrix to Some Canonical Form}

We now state the proposition

(42) Let $D$ be a non empty set, $m, n, i, j$ be elements of $\mathbb{N}$, and $A$ be a matrix over $D$ of dimension $m \times n$. Then $\operatorname{Swap}(A, i, j)$ is a matrix over $D$ of dimension $m \times n$.

Let us consider $K$, let $n$ be an element of $\mathbb{N}$, and let $i_{0}$ be a natural number. The functor $\operatorname{SwapDiagonal}\left(K, n, i_{0}\right)$ yields a square matrix over $K$ of dimension $n$ and is defined as follows:

(Def. 3) $\operatorname{SwapDiagonal}\left(K, n, i_{0}\right)=\operatorname{Swap}\left(I_{K}^{n \times n}, 1, i_{0}\right)$.

Next we state a number of propositions:

(43) Let $n$ be an element of $\mathbb{N}, i_{0}$ be a natural number, and $A$ be a square matrix over $K$ of dimension $n$. Suppose $1 \leq i_{0} \leq n$ and $A=$ SwapDiagonal $\left(K, n, i_{0}\right)$. Let $i, j$ be natural numbers. Suppose $1 \leq i \leq n$ and $1 \leq j \leq n$. Suppose $i_{0} \neq 1$. Then

(i) if $i=1$ and $j=i_{0}$, then $A_{i, j}=1_{K}$,

(ii) if $i=i_{0}$ and $j=1$, then $A_{i, j}=1_{K}$,

(iii) if $i=1$ and $j=1$, then $A_{i, j}=0_{K}$,

(iv) if $i=i_{0}$ and $j=i_{0}$, then $A_{i, j}=0_{K}$, and

(v) if $i \neq 1$ and $i \neq i_{0}$ or $j \neq 1$ and $j \neq i_{0}$, then if $i=j$, then $A_{i, j}=1_{K}$ and if $i \neq j$, then $A_{i, j}=0_{K}$.

(44) Let $n$ be an element of $\mathbb{N}, A$ be a square matrix over $K$ of dimension $n$, and $i$ be a natural number. If $1 \leq i \leq n$, then $(\operatorname{SwapDiagonal}(K, n, 1))_{i, i}=$ $1_{K}$.

(45) Let $n$ be an element of $\mathbb{N}, A$ be a square matrix over $K$ of dimension $n$, and $i, j$ be natural numbers. If $1 \leq i \leq n$ and $1 \leq j \leq n$, then if $i \neq j$, then $(\operatorname{SwapDiagonal}(K, n, 1))_{i, j}=0_{K}$. 
(46) Let given $K, n, i_{0}$ be elements of $\mathbb{N}$, and $A$ be a square matrix over $K$ of dimension $n$. Suppose that

(i) $1 \leq i_{0}$,

(ii) $i_{0} \leq n$,

(iii) $i_{0}=1$, and

(iv) for all natural numbers $i, j$ such that $1 \leq i \leq n$ and $1 \leq j \leq n$ holds if $i=j$, then $A_{i, j}=1_{K}$ and if $i \neq j$, then $A_{i, j}=0_{K}$.

Then $A=\operatorname{SwapDiagonal}\left(K, n, i_{0}\right)$.

(47) Let given $K, n, i_{0}$ be elements of $\mathbb{N}$, and $A$ be a square matrix over $K$ of dimension $n$. Suppose that

(i) $1 \leq i_{0}$

(ii) $i_{0} \leq n$,

(iii) $\quad i_{0} \neq 1$, and

(iv) for all natural numbers $i, j$ such that $1 \leq i \leq n$ and $1 \leq j \leq n$ holds if $i=1$ and $j=i_{0}$, then $A_{i, j}=1_{K}$ and if $i=i_{0}$ and $j=1$, then $A_{i, j}=1_{K}$ and if $i=1$ and $j=1$, then $A_{i, j}=0_{K}$ and if $i=i_{0}$ and $j=i_{0}$, then $A_{i, j}=0_{K}$ and if $i \neq 1$ and $i \neq i_{0}$ or $j \neq 1$ and $j \neq i_{0}$, then if $i=j$, then $A_{i, j}=1_{K}$ and if $i \neq j$, then $A_{i, j}=0_{K}$.

Then $A=\operatorname{SwapDiagonal}\left(K, n, i_{0}\right)$.

(48) Let $A$ be a square matrix over $K$ of dimension $n$ and $i_{0}$ be an element of $\mathbb{N}$. Suppose $1 \leq i_{0} \leq n$. Then

(i) for every $j$ such that $1 \leq j \leq n$ holds $\left(\operatorname{SwapDiagonal}\left(K, n, i_{0}\right) \cdot A\right)_{i_{0}, j}=$ $A_{1, j}$ and $\left.\operatorname{SwapDiagonal}\left(K, n, i_{0}\right) \cdot A\right)_{1, j}=A_{i_{0}, j}$, and

(ii) for all $i, j$ such that $i \neq 1$ and $i \neq i_{0}$ and $1 \leq i \leq n$ and $1 \leq j \leq n$ holds $\left(\operatorname{SwapDiagonal}\left(K, n, i_{0}\right) \cdot A\right)_{i, j}=A_{i, j}$.

(49) For every element $i_{0}$ of $\mathbb{N}$ such that $1 \leq i_{0} \leq n$ holds $\operatorname{SwapDiagonal}\left(K, n, i_{0}\right)$ is invertible and $\left(\operatorname{SwapDiagonal}\left(K, n, i_{0}\right)\right)^{\smile}=$ $\operatorname{SwapDiagonal}\left(K, n, i_{0}\right)$.

(50) For every element $i_{0}$ of $\mathbb{N}$ such that $1 \leq i_{0} \leq n$ holds (SwapDiagonal $\left.\left(K, n, i_{0}\right)\right)^{\mathrm{T}}=\operatorname{SwapDiagonal}\left(K, n, i_{0}\right)$.

(51) Let $A$ be a square matrix over $K$ of dimension $n$ and $j_{0}$ be an element of $\mathbb{N}$. Suppose $1 \leq j_{0} \leq n$. Then

(i) for every $i$ such that $1 \leq i \leq n$ holds $\left(A \cdot \operatorname{SwapDiagonal}\left(K, n, j_{0}\right)\right)_{i, j_{0}}=$ $A_{i, 1}$ and $\left(A \cdot \operatorname{SwapDiagonal}\left(K, n, j_{0}\right)\right)_{i, 1}=A_{i, j_{0}}$, and

(ii) for all $i, j$ such that $j \neq 1$ and $j \neq j_{0}$ and $1 \leq i \leq n$ and $1 \leq j \leq n$ holds $\left(A \cdot \operatorname{SwapDiagonal}\left(K, n, j_{0}\right)\right)_{i, j}=A_{i, j}$.

(52) Let $A$ be a square matrix over $K$ of dimension $n$. Then $A=0_{K}^{n \times n}$ if and only if for all $i, j$ such that $1 \leq i \leq n$ and $1 \leq j \leq n$ holds $A_{i, j}=0_{K}$. 


\section{Left/Right InVERTIBILITy And InvertibiLity}

The following four propositions are true:

(53) Let $A$ be a square matrix over $K$ of dimension $n$. Suppose $A \neq 0_{K}^{n \times n}$. Then there exist square matrices $B, C$ over $K$ of dimension $n$ such that

(i) $B$ is invertible,

(ii) $C$ is invertible,

(iii) $(B \cdot A \cdot C)_{1,1}=1_{K}$,

(iv) for every $i$ such that $1<i \leq n$ holds $(B \cdot A \cdot C)_{i, 1}=0_{K}$, and

(v) for every $j$ such that $1<j \leq n$ holds $(B \cdot A \cdot C)_{1, j}=0_{K}$.

(54) Let $A, B$ be square matrices over $K$ of dimension $n$. Suppose $B \cdot A=$ $I_{K}^{n \times n}$. Then there exists a square matrix $B_{2}$ over $K$ of dimension $n$ such that $A \cdot B_{2}=I_{K}^{n \times n}$.

(55) Let $A$ be a square matrix over $K$ of dimension $n$. Then the following statements are equivalent

(i) there exists a square matrix $B_{1}$ over $K$ of dimension $n$ such that $B_{1} \cdot A=$ $I_{K}^{n \times n}$

(ii) there exists a square matrix $B_{2}$ over $K$ of dimension $n$ such that $A \cdot B_{2}=$ $I_{K}^{n \times n}$.

(56) For all square matrices $A, B$ over $K$ of dimension $n$ such that $A \cdot B=$ $I_{K}^{n \times n}$ holds $A$ is invertible and $B$ is invertible.

\section{REFERENCES}

[1] Grzegorz Bancerek and Krzysztof Hryniewiecki. Segments of natural numbers and finite sequences. Formalized Mathematics, 1(1):107-114, 1990.

[2] Czesław Byliński. Finite sequences and tuples of elements of a non-empty sets. Formalized Mathematics, 1(3):529-536, 1990.

[3] Czesław Byliński. Functions and their basic properties. Formalized Mathematics, 1(1):5565, 1990.

[4] Czesław Byliński. Partial functions. Formalized Mathematics, 1(2):357-367, 1990.

[5] Shigeru Furuya. Matrix and Determinant. Baifuukan (in Japanese), 1957.

[6] Felix R. Gantmacher. The Theory of Matrices. AMS Chelsea Publishing, 1959.

[7] Katarzyna Jankowska. Matrices. Abelian group of matrices. Formalized Mathematics, 2(4):475-480, 1991.

[8] Eugeniusz Kusak, Wojciech Leończuk, and Michał Muzalewski. Abelian groups, fields and vector spaces. Formalized Mathematics, 1(2):335-342, 1990.

[9] Takaya Nishiyama and Yasuho Mizuhara. Binary arithmetics. Formalized Mathematics, 4(1):83-86, 1993.

[10] Andrzej Trybulec. Binary operations applied to functions. Formalized Mathematics, 1(2):329-334, 1990.

[11] Wojciech A. Trybulec. Binary operations on finite sequences. Formalized Mathematics, 1(5):979-981, 1990.

[12] Wojciech A. Trybulec. Groups. Formalized Mathematics, 1(5):821-827, 1990.

[13] Wojciech A. Trybulec. Vectors in real linear space. Formalized Mathematics, 1(2):291-296, 1990.

[14] Zinaida Trybulec. Properties of subsets. Formalized Mathematics, 1(1):67-71, 1990.

[15] Hiroshi Yamazaki, Yoshinori Fujisawa, and Yatsuka Nakamura. On replace function and swap function for finite sequences. Formalized Mathematics, 9(3):471-474, 2001. 
[16] Xiaopeng Yue, Xiquan Liang, and Zhongpin Sun. Some properties of some special matrices. Formalized Mathematics, 13(4):541-547, 2005.

[17] Katarzyna Zawadzka. The sum and product of finite sequences of elements of a field. Formalized Mathematics, 3(2):205-211, 1992.

[18] Katarzyna Zawadzka. The product and the determinant of matrices with entries in a field. Formalized Mathematics, 4(1):1-8, 1993.

Received April 2, 2008 